\title{
LIBRI E ARTIGIANI DEL LIBRO: LE RACCOMANDAZIONI DEI GIURISTI MUSULMANI (XIV SECOLO)*
}

\author{
Giovanni Canova \\ UNIVERSITÀ DEGLI STUDI DI NAPOLI “L'ORIENTALE"
}

The paper deals with the comments 14 th-century jurists, Ibn Ğama $\bar{a}^{c} a$, Ibn al-H̄āğğ and al-Subkī made about books and bookmaking, which highlight their ambivalent attitude towards the craftsmanship of those involved in the production of books. While extolling the merits of papermakers, scribes and bookbinders in connection with the production of religious books, the jurists urged these craftsmen to proceed in strict compliance with the Islamic code. Copying and binding certain kinds of books, such as folk romances, was severely censured or even prohibited. Some suggestions aiming at the prevention of fraud and illicit behaviour were also included in sections of legal treatises dealing with the crafts of book-production.

È ben noto il prestigio del libro nel mondo islamico: il 'Libro' per eccellenza è lo stesso Corano. In un importante saggio sulle biblioteche abbasidi, Olga Pinto scrive che 'presso pochi popoli il culto del libro e della tradizione letteraria ed erudita ha avuto tanta importanza in tutta quanta la vita spirituale e culturale quanta ne ha avuta presso gli Arabi'. ${ }^{1}$ Tre erano state, secondo lo storico al-Qalqašandī (m. 821/1418) le principali biblioteche: quella abbaside a Baghdad, quella fatimide al Cairo e quella omayyade a Cordova. ${ }^{2}$ Particolarmente dense sono le pagine dedicate dallo storico Ibn Hualdūn (m. 808/1406) alla trasmissione del sapere tramite la scrittura e la produzione di libri, la cui perfezione o decadenza sono in stretta correlazione con l'analoga condizione della società urbana. ${ }^{3}$ I testi ci lasciano intravedere un'intensa attività di

\footnotetext{
* Questo saggio riprende una serie di studi sulle professioni legate al libro arabo e intende presentare una riflessione sintetica sulla posizione dei giuristi in un periodo chiave per la produzione di libri: l'epoca mamelucca.

1 Cfr. Pinto, 'Le Biblioteche degli Arabi', 140.

2 Cfr. J. Pedersen, The Arabic Book, 113. Si veda inoltre A. Grohmann, 'Bibliotheken und Bibliophilen im islamischen Orient', in Festschrift der Nationalbibliothek in Wien, 431-42; L. Capezzone, La trasmissione del sapere nell'Islam medievale, cap. vi.

3 Cfr. Ibn Haldūn, Muqaddima, 2: 889 (The Muqaddimah, 2: 392).
} 
copisti, rilegatori, decoratori, mercanti di libri, bibliofili sia nell'Oriente che nell'Occidente islamico. ${ }^{4}$

Nella Baghdad del X secolo, all'epoca di al-Ya ${ }^{c} q u \bar{b} \overline{1}$, operavano più di cento librai e già esisteva quello che noi definiremmo il mercato antiquario. $^{5}$ Il commercio librario si svolgeva in genere nelle parti più nobili del mercato, presso la principale moschea cattedrale delle grandi città. ${ }^{6}$ Nella pagina dedicata al sūq al-kutubiyyīn del Cairo, al-Maqrīzī (m. 845/1441) sottolinea come questo costituisse un luogo privilegiato di incontro tra i dotti, l'unico degno di essere frequentato assieme a quello del mercato delle armi. Assieme a kutubī, 'libraio', si trova nei testi forse ancora più frequentemente il vocabolo warrāq, con il molteplice significato di fabbricante di carta in senso stretto, cartolaio, venditore di libri, ma anche rilegatore, copista o scriba. ${ }^{7}$ Analoghe considerazioni sono possibili per wirāqa, 'l'industria del libro'. Famoso warrāq fu Ibn al-Nadīm (m. 388/998), l'autore del Fihrist, il grande repertorio suddiviso per materia di tutti i libri in arabo noti al suo tempo.

L'VIII secolo dell'egira (XIV sec.) sembra essere il periodo in cui giuristi e ulema più hanno scritto e più si sono occupati delle attività concernenti la produzione di libri. Questo è probabilmente dovuto al fatto che l'epoca mamelucca aveva visto fiorire pratiche di lavoro, comportamenti, letterature, espressioni artistiche che mal si conciliavano con le accorate raccomandazioni degli uomini di religione, custodi della

4 Sul manoscritto arabo-islamico si veda A. Gacek, The Arabic Manuscript Tradition; Id., Supplement; Id., Arabic Manuscripts. A Vademecum for Readers; e inoltre J. Pedersen, The Arabic Book; A.F. Sayyid, al-Kitāb al- ${ }^{c}$ arabī almahtūt wa- ${ }^{c}$ ilm al-mahtūtạț; F. Déroche e F. Richard (eds), Scribes et manuscrits du Moyen Orient; F. Déroche, Manuel de codicologie des manuscrits en écriture arabe; Id., Le livre manuscript arabe; S.S. Blair, Islamic Calligraphy.

5 A. von Kremer, Kulturgeschichte des Orients unter den Chalifen, 2: 310. L'autore dedica pagine interessanti all'industria della carta, al libro e alla sua decorazione, al commercio librario, alla bibliofilia, all'arte dello scriba (304-15).

6 Sull'organizzazione della città araba medievale e sulle attività artigianali e mercantili, si veda, tra l'altro: G.E. von Grunebaum, 'The Structure of the Muslim Town, in Islam. Essays in the Nature and Growth of a Cultural Tradition, 141-58; L. Massignon, 'Les corps de métiers et la cité islamique', in Opera minora, 1: 369-84; I.M. Lapidus, Muslim Cities in the Later Middle Ages; A.H. Hourani S.M. Stern (eds.), The Islamic City. A Colloquium; E. Ashtor, Storia sociale ed economica del Vicino Oriente nel Medioevo, trad. it.; B. Scarcia Amoretti, Un altro Medioevo. Il quotidiano nell'Islam, cap. iii.

7 Cfr. Hasan al-Bāšāā, al-Funūn al-islāmiyya, 3: 1321; A. Gacek, The Arabic Manuscript Tradition, 150. 
stretta ortodossia. Queste pratiche si configuravano come innovazioni biasimevoli (bida ${ }^{c}$, sing. bid $\left.{ }^{c} a\right){ }^{8}$ Le varie scuole giuridiche concordano su questo atteggiamento. Spiccano i nomi del malikita Muhammad b. alḤāğğ al-Fāsī al- ${ }^{\mathrm{c}}$ Abdarī (m. 737/1336) e degli shaficiti Tāğ al-Dīn alSubkī (m. 771/1370) e Ibn Ğamā ${ }^{\mathrm{c} a}$ (m. 733/1333). ${ }^{9}$ Essi fondano le loro argomentazioni sui concetti di mital 'modelli di comportamento', adab 'corretto modo di agire', niyya 'buon proposito'.

In quanto segue presento le parti più significative dei tre trattati, precedute da alcune informazioni biografiche utili per inquadrare i loro autori. L'insieme delle 'raccomandazioni', spesso molto minuziose in relazione all'opera dello scriba, si può riassumere in due concetti fondamentali: 1) l'artigiano del libro deve comportarsi in tutte le sue azioni seguendo una stretta etica islamica; 2) deve operare possedendo la necessaria perizia tecnica e con un alto livello di professionalità.

\section{1) Taqì al-Dīn e Tāğ al-Dīn al-Subkī}

Taqī l-Dīn al-Subkī (m. 756/1355), gran $q \bar{a} d \bar{l} \bar{\imath}$ di Damasco e predicatore nella moschea degli Omayyadi, si occupò di libri e di biblioteche, trasmettendoci il documento waqf della al-Ašrafiyya. Le informazioni riportate nella sua raccolta di fatwa costituiscono un'importante attestazione per la conoscenza dell'organizzazione e del funzionamento della biblioteca:

Si versino mensilmente al bibliotecario 18 dirham. Egli avrà cura del restauro [delle legature] dei libri e ne renderà conto al soprintendente (näzir) o al suo sostituto, al fine di stanziare le somme necessarie dalle rendite della fondazione. Agirà nello stesso modo, qualora se ne presenti la necessità, per correggere il libro o collazionarlo... Interverrà per la fornitura di carta, degli strumenti di scrittura quali penne, calamai, supporti di libri (karāriss) etc., che metterà al servizio di coloro che operano nel grande $\bar{i} w \bar{a} n$ o nella sala di fronte, copiando [opere] di hadīi e di scienze ausiliarie, passi del Corano e commentari. Interverrà per le necessità di quelli che trascrivono nelle sedute di dettatura $\left(i m l \bar{a}^{\supset}\right)$ e di coloro che scrivono libri o raccolte di certificati (istiğăza). Egli accorderà tuttavia il suo favore solo a quelli che copiano per i propri studi e non per ricavarne un guadagno e un profitto pecuniario. Il soprintendente può fare copiare o acquistare, per

8 Sui kutub al-bidac si veda in particolare Fierro, 'The Treatises against Innovations', e bibliografia citata.

9 Un paio di secoli dopo, ${ }^{c}$ Abd al-Bāsit al- ${ }^{c}$ Almawī (m. 981/1573), šafic ita, riprende il testo di Ibn Ğama $\bar{a}^{c} a$ apportando integrazioni e chiarimenti. Un fondamentale studio su questo autore è stato pubblicato da F. Rosenthal, The Technique and Approach of Muslim Scholarship, 7-18. 
conto della fondazione, i libri e i fascicoli necessari, che egli costituirà in waqf a somiglianza dei fondi già esistenti. ${ }^{10}$

Chiara testimonianza delle preoccupazioni dei dottori dell'Islam si trova nei capitoli sugli artigiani del libro in un'opera del figlio di Taqī l-Dīn alSubkī, Tāă al-Dīn (m. 771/1370), ${ }^{11}$ l'autore delle Tabaqāt al-šăficiyya, ('Classi degli Shaficiti'). Tra i suoi numerosi scritti figura un trattato di etica del lavoro, il Kitāb $M u^{c} \bar{\imath} d$ al-nic am wa-mubìd al-niqam, ('Il restauratore dei favori e l'estirpatore dei castighi'). ${ }^{12}$ Secondo l'autore, la comunità musulmana deve distogliersi dagli interessi terreni, rendendo grazie a Dio nel proprio lavoro quotidiano. L'opera è strutturata in centotredici mițâl, 'modelli di comportamento' cui devono attenersi i vari componenti della società, dal califfo, al sultano e ai dignitari, via via fino ai lavoratori più umili. ${ }^{13}$

Tāğ al-Dīn al-Subkī assume un atteggiamento censorio nei confronti degli artigiani del libro, dal copista fino al rilegatore e al decoratore. Nella sua requisitoria egli opera una netta distinzione tra gli scritti profani e le opere religiose: non solo condanna i libri 'eretici' o licenziosi, ma anche le popolari biografie leggendarie degli antichi eroi arabi, quali ${ }^{c}$ Antara $b$. Šaddād, con la inquietante motivazione che 'Dio non ne trae vantaggio'. Anche se è lecito chiedersi quale sia stata l'efficacia di simili anatemi, alSubkī manifesta una preoccupazione condivisa tra gli ${ }^{c} u l a m \bar{a}^{p} \cdot{ }^{14}$

Il rapporto tra autore o committente e copista non sempre è privo di diffidenza e di contrasti, tanto da richiedere talora un responso giuridico. Anche i rilegatori dovrebbero limitare la propria opera a libri di carattere strettamente religioso; solo il Corano dovrebbe essere oggetto di doratura.

${ }^{10}$ La citazione è tratta da Eche, Les Bibliothèques arabes, 214-15. Sulla famiglia al-Subkī, si veda la voce curata da J. Schacht-[C.E. Bosworth], in $E I^{2}$, 9: $776-8$

11 Per notizie sulla vita e sulle opere di Tāŏ al-Dīn al-Subkī si veda GAL 2: 89-90.

${ }^{12}$ Ed. Muḥammad 'Alī al-Nağğāàr, Abū Zayd Šalabī e Muḥammad Abū 1'Uyūn, 1948, che corregge l'edizione D.W. Myhrman del 1908.

$13 \mathrm{Si}$ vedano le osservazioni di M. Shatzmiller, Labour in the Medieval Islamic World, 77-8, $387 \mathrm{sgg}$.

${ }^{14}$ Forse al-Subkī riteneva che il pathos che caratterizza queste narrazioni riflettesse più lo spirito della Ğāhiliyya che quello dell'Islam; o forse temeva la popolarità del cantastorie, che aveva presa sul pubblico più del predicatore (ricordiamo che egli stesso era stato hațīb alla Moschea degli Omayyadi di Damasco). I riferimenti all'epica araba sono stati da me esaminati, assieme a quelli di altri autori, nel saggio 'Critical Attitudes Toward Arabic Folk Epics' (2005). 
L'accorato appello di Tā̆g al-Dīn al-Subkī sembra però aver avuto, fortunatamente, un'influenza limitata sull'evoluzione dell'arte del libro islamico.

\section{Dal Kitāb $M u^{c} \bar{\imath} d$ al-nic ${ }^{c}$ am wa-mubìd al-niqam: ${ }^{15}$}

lxxix. Lo scriba/copista (al-nāsih)

È suo dovere non copiare nulla in fatto di libri fuorvianti, come quelli degli eretici e dei settari, e astenersi dal copiare libri di cui Dio non trae vantaggio, come la Sira di ${ }^{c}$ Antara e altre simili di fantasia, che costituiscono solo una perdita di tempo e dei quali la religione non ha alcun bisogno. Lo stesso vale per i libri dei libertini e per ciò che essi scrivono sulle modalità del coito, sulle qualità delle bevande inebrianti e su ogni altra cosa che istighi [a compiere] azioni proibite. Mettiamo in guardia i copisti da tutto ciò: la vita terrena li trae in errore. Spesso colui che commissiona di scrivere queste cose offre un compenso maggiore di quello che chiede libri di scienza. È tuttavia opportuno che il copista 'non svenda la sua religione per i beni del mondo'.

Ci sono copisti che non provano timor di Dio e scrivono affrettatamente, omettendo passi del libro per fretta di finirlo, malgrado il compenso sia stato pattuito per la copiatura integrale. Queste persone sono dei traditori nei confronti di Dio, dell'autore e di chi li ricompensa per il loro lavoro.

I giuristi hanno detto: se [al copista] viene commissionato di scrivere un testo e commette degli errori, o di scriverlo in arabo e invece lo fa in lingua diversa ( $\left.{ }^{c} a \breve{g} a m i y y a\right)$, o il contrario, egli è responsabile della perdita della carta e non gli spetta alcun compenso. Al-Nawawī [m. 676/1277] diede il seguente responso - e quanto ha ricordato al-Ġazzālī [m. 505/1111] nelle

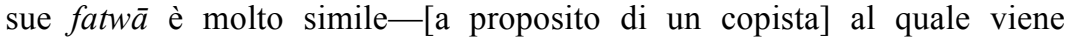
commissionata la copiatura di un libro ed egli cambia la sequenza dei capitoli: se è possibile rimettere in ordine le varie parti del testo, ad esempio se il libro si compone di dieci capitoli e il copista ha scritto il primo [ponendolo] separato alla fine, ha diritto all'ammontare dell'importo pattuito; altrimenti non ha diritto a nulla. (...).

lxxx. Il fabbricante di carta (al-warrāq $)$

Si tratta di uno dei più eccellenti lavori, perché su di esso si basa la scrittura dei Corani, dei libri di scienza, dei documenti e dei contratti della gente. Che colui che svolge questa attività, per grazia di Dio, favorisca la ricerca della scienza e delle altre [discipline]; dia la preferenza a chi egli sa che acquista la carta per scrivere libri di scienza, ma si rifiuti di venderla a chi sa che scriverà ciò che non si conviene: scritti eretici ed eterodossi, testimonianze e atti giudiziari falsi e simili.

15 Ed. Cairo, Maktabat al-Hูānğ̄i, $1996^{3}$. 
1xxxi. The bookbinder (al-muğallid $)^{16}$

È suo dovere comportarsi come il copista e il fabbricatore di carta.

lxxxii. Il decoratore-doratore (al-mudahhib)

È suo dovere dorare solamente copie del Corano. Sono note le divergenze tra $\mathrm{i}$ dotti sulla decorazione del Corano con oro. Al-Rāfi ${ }^{c} \overline{1}$ e al-Nawawī sostengono che c'è differenza se esso appartenga a una donna, e in tal caso è ammessa, oppure a un uomo, e allora è vietata. Secondo noi è preferibile ammetterne la liceità senza restrizioni. Per quanto riguarda libri diversi dal Corano, i giuristi concordano nel non considerare lecita la decorazione con oro.

lvii. Il conservatore di libri (hāzin al-kutub)

È suo dovere conservare i libri, riassestarli (tarmīmuhā) quando sono scompaginati, rilegarli (habkuhā) qualora se ne presenti la necessità. Egli deve essere accorto nel darli a coloro che non li rispettano, ma prodigo nei confronti di quelli che ne abbisognino; è inoltre opportuno che favorisca nel prestito i poveri, i quali hanno difficoltà nel procacciarsi libri, piuttosto che i ricchi. Frequentemente la persona che li ha dati in lascito (wāqif) pone la condizione che il libro sia consegnato solo dietro il versamento di una cauzione di valore corrispondente. Si tratta di una condizione giusta e da tenere in considerazione: il conservatore non deve concedere prestiti se non dietro cauzione. (...)

civ. I sensali/commissionari (al-dallālūn)

Fra questi vi è il commissionario di libri. Egli deve astenersi dal vendere libri religiosi a persone che, come risaputo, li rovinerebbero o li leggerebbero [solo] per criticarli e recare loro discredito. E neppure devono vendere opere di eretici e settari, né libri di astrologi e storie inventate come la Sïra di ${ }^{\mathrm{c}}$ Antara e altri. Non gli è lecito vendere a un miscredente né il Corano né libri di Tradizioni [del Profeta] e neppure di diritto.

\section{2) Ibn $\check{G} a m \bar{a}^{c} a$}

Badr al-Dīn Muḥammad b. Ibrāhīm b. Sa ${ }^{c} d$ Allāh Ibn Ğamāca al-Kinān̄̄ al-Hamawī al-Šăfic ${ }^{\top} \overline{1}$ (m. 733/1333), membro di un'importante famiglia di giuristi siriani, raggiunse un'alta posizione nell'impero mamelucco. A Gerusalemme fu predicatore e imam nella moschea al-Aqșā, e successivamente $q \bar{a} d \underline{\imath}$ della città. Alla morte del gran qāộ d'Egitto (690/1291), il sultano al-Ašraf Halīl lo nominò al suo posto, assegnandogli anche la carica di šayh̆ al-šuyūh, capo delle confraternite. In seguito alle vicissitudini dei sultani che si succedettero nel regno, fu dimesso dal suo incarico al Cairo, operò per un periodo come gran qạḍ̄ a

16 Hasan al-Bāšā, al-Funūn al-islāmiyya, 3: 1023-5, riporta i nomi di molti artigiani che hanno rese illustri l'arte della legatura e della decorazione dei libri islamici nelle varie epoche, in particolare quella mamelucca. 
Damasco; successivamente fece ritorno in Egitto dove riprese la sua attività. ${ }^{17}$

Tra le sue opere figurano un trattato sui diritti e doveri del sultano (Tahrīr al-ahkām fì tadbīr ahl al-islām), vari libri sulle tradizioni del Profeta e altre opere minori. ${ }^{18}$ La Tadkirat al-sāmi ${ }^{c}$ wa-l-mutakallim fi adab al- "álim wa-l-mutacallim ('Memoria per chi ascolta e chi parla in merito alle buone maniere del dotto e del discente') è un trattato sulla trasmissione del sapere religioso e sull'educazione nella madrasa. L'autore insiste sul concetto di $\bar{a} d \bar{a} b$, 'corretto comportamento, buone maniere', ${ }^{19}$ quella 'bontà di comportamento (husn al-adab) che con $\mathrm{i}$ suoi meriti è testimonianza di retta via e intelligenza'. ${ }^{20}$ L'opera è articolata in cinque ampie sezioni, i cui titoli ben evidenziano il contenuto: i. 'Sul merito della scienza e dei dotti, del suo insegnamento e del suo apprendimento'; ii. 'Sulle buone maniere del dotto verso se stesso e nel rispetto del suo studente e dello studio'; iii. 'Sulle buone maniere del discente'; iv. 'Sulle buone maniere con i libri'; v. 'Sulle buone maniere di coloro che frequentano le scuole'.

Nella traduzione che segue, del iv capitolo, ${ }^{21}$ vengono ripresi i sottotitoli aggiunti per maggiore chiarezza dall'editore al-Nadwī. ${ }^{22}$

Dalla Tadkirat al-sāmi ${ }^{c}$. Libri e corretto comportamento:

Cap. iv: Sul corretto comportamento concernente i libri, che costituiscono lo strumento [per trasmettere] la scienza, e ciò che vi si riferisce in merito alla loro edizione, vocalizzazione, trasferimento, collocazione, e inoltre acquisto, prestito, copia etc. Il capitolo comprende dieci sezioni:

17 Si veda al-Șafadī, Das biographische Lexicon, ed. S. Dedering, 2: 18-20, $\mathrm{n}^{\circ}$ 268; al-Subkī, al-Tabaqāt al-šăfi $f^{c}$ iyya al-kubrāa, 5: 230-3; K.S. Salibi, The


Ibn Djama $^{c} a$, in $E I^{2}, 3: 771-2$.

18 GAL, 2: 74-5, GAL-S, 2: 80-1; al-Ziriklī, al-Aclām, 6: 188-9.

19 L'evoluzione del termine $a d a b$, pl. àdāb, dal significato antico di 'abitudine, norma di condotta, costume', a quello successivo di 'buona qualità dell'anima, buona educazione, urbanità, cortesia', e infine a quello di 'letteratura', 'belles lettres', è stata analizzata in particolare da K. Vollers e C.A. Nallino. Si veda la sintesi di F. Gabrieli, $A d a b$, in $E I^{2}, 1: 180-1$ e il saggio di M. Cassarino, 'Ancora sulla definizione di $a d a b$ '.

${ }^{20}$ Tad $\underline{d}$ kirat al-sāmi ${ }^{c}, 13$.

21 Ibid., 151-72.

${ }^{22}$ L'edizione è stata effettuata sulla base del codice migliore (Gotha, ms. ar. 162, del 682/1275), con l'aggiunta di copiose note; l'opera è stata riproposta dalla Dār al-Kutub al-'ilmiyya di Beirut una prima volta in edizione anastatica (1974) e successivamente in una nuova veste tipografica (2005). 
Sezione i: [Sollecitudine degli studenti nel procurarsi i libri] ${ }^{23}$

È opportuno che 'colui che cerca la scienza' (tâ̆lib al- ${ }^{c} i l m$, lo studente/lo studioso) sia sollecito nel procurarsi i libri di cui necessita, per quanto gli è possibile acquistandoli, altrimenti dietro compenso o in prestito, poiché costituiscono lo strumento per giungere alla scienza. Tuttavia averne in gran numero non significa necessariamente possederla né comprenderla, come ritengono molti che si atteggiano a esperti di giurisprudenza e hadit. Sono giuste le parole di chi ha detto:

Se non tieni a mente [ $=$ non studi] con consapevolezza il tuo accumular libri non ti giova.

Se [lo studente / lo studioso] è in grado di acquistare i libri, non si impegni nella loro copiatura. Quest'ultima non è auspicabile per il tempo che richiede, a meno che egli non possa farlo, non essendo in grado di pagare il loro prezzo né il compenso per farseli copiare. Non si preoccupi eccessivamente di una bella scrittura, ma piuttosto della correttezza di quanto scrive. Non chieda in prestito un libro se ha la possibilità di acquistarlo o averlo dietro compenso.

Sezione ii: [Presa in prestito dei libri in caso di necessità] ${ }^{24}$

È auspicabile il prestito di libri a condizione che non subisca un danno chi li presta o chi li prende a prestito. Ad alcuni ripugna dare a prestito, ma il primo comportamento [cioè il prestare libri] è migliore, poiché in ciò vi è aiuto alla scienza e in sé è un'azione meritoria degna di ricompensa. (...) È opportuno che colui che chiede in prestito un libro ringrazi il prestatore e lo ricompensi con una buona azione. Non si prolunghi il possesso [del libro] senza necessità, anzi sia restituito quando è terminata [la lettura o copiatura]. Non si trattenga ulteriormente quando non ce n'è più bisogno $o$ il suo proprietario lo richieda. Non è lecito introdurre correzioni senza permesso del proprietario.

Non si appongano note o scritte nello spazio vuoto delle pagine iniziali (fawātih) o finali (hawātim), a meno che il proprietario del libro non sia d'accordo. Allo stesso modo deve comportarsi chi trascrive hadīt quando riporta la parte che ha udito o trascritto. Non ne faccia una brutta copia, non lo presti a terze persone né lo dia senza necessità anche se è lecito, e neppure lo copi senza permesso del proprietario.

Se il libro fa parte di una donazione pia (waqf), lasciato in uso a chi ne può trarre utilità, senza che ci siano particolari condizioni, non c'è nulla di male nel copiarlo, con la riserva che può introdurre eventuali correzioni solo chi ne ha la competenza; è in ogni caso bene che venga chiesto

23 Cfr. al- ${ }^{\mathrm{c}}$ Almawī, al-Mu ${ }^{c} \bar{\imath} d$ fi $a d a b$ al-mufìd, 251; Rosenthal, The Technique, 8-9.

${ }^{24}$ Cfr. al- ${ }^{\mathrm{c}}$ Almawī, al-Mu ${ }^{c} \bar{\imath} d$ fi $a d a b$ al-mufìd, 251-3; Rosenthal, The Technique, 9-10. 
preventivamente il permesso al supervisore. Se, ottenuto questo permesso, lo copia, non scriva su di esso o su una pagina aggiunta o posta sopra lo scritto. Non ponga il calamaio sopra il libro, né faccia passare il calamo intriso di inchiostro sopra la pagina scritta [con il pericolo di macchiarla]. Un poeta ha detto:

$\mathrm{O}$ tu che prendi a prestito un libro da me fammi contento [trattando bene il mio libro]

come vorresti esserlo tu [se fossi tu a prestarmelo].

Sezione iii [Come disporre i libri durante al consultazione] ${ }^{25}$ Quando si copia o si sta consultando un libro, questo non deve venire posto dispiegato sul pavimento, ma tra due libri o altri due oggetti, o [preferibilmente] posato sul leggio (kursī al-kutub), ${ }^{26}$ al fine di non affrettare la rottura del filo di cucitura. Se si ripone il libro in una pila, ciò non deve avvenire sul supporto menzionato né su un palchetto di legno (taht hašab) o simili. La cosa migliore è mantenere il libro sollevato dal suolo, affinché non si bagni o assorbi umidità.

Se il libro viene posto su un supporto di legno o simili, è opportuno inserire sopra e sotto qualcosa che impedisca danni alla pelle della coperta; allo stesso modo, i libri devono venire protetti dal contatto con un muro o altro appoggio. Il retto comportamento (adab) nel riporre i libri tiene in considerazione le scienze ( $\left.{ }^{c} u l u \bar{m}\right)$ [che vi sono trattate], la loro importanza, il loro autore, la loro eccellenza.

Il libro più 'nobile' deve venir collocato più in alto rispetto a tutti gli altri, tenendo in conto la suddivisione in classi (tadrĭğ). Se c'è un Corano (mușhaf) deve stare sopra tutti; meglio se inserito in un contenitore con un appiglio da appendere a un chiodo o un picchetto su una parete pulita, in mezzo alla stanza dove ha luogo la riunione. Successivamente vengono, nell'ordine, i libri di tradizioni genuine del Profeta, come al-Sahịh di Muslim, quindi il commentario del Corano, il commentario del hadīt, i fondamenti della religione (ușūl al-dīn) e della giurisprudenza (ușül al-fiqh), opere di grammatica e sintassi, infine le 'poesie degli arabi' ( $a \check{s}^{c} \bar{a} r$ al- ${ }^{c} a r a b$ ) e i trattati di metrica ( ${ }^{c} a r \bar{u}()$ ). Se ci sono due libri dello stesso soggetto, viene posto in alto quello che contiene maggiormente parti del Corano o di hadīt; se in ciò non vi è differenza, è da tenere in considerazione l'importanza dell'autore $\mathrm{e}$, in secondo luogo, la scrittura più antica o il maggior utilizzo da parte di dotti e persone pie, quindi il testo più corretto.

25 Cfr. al- ${ }^{\mathrm{c}}$ Almawī, al-Mu ${ }^{c} \bar{\imath} d$ fi adab al-mufìd, 253-4; Rosenthal, The Technique, $10-11$.

${ }^{26}$ Il tradizionale leggio di legno a forma di X. Si veda Gacek, Arabic Manuscripts. A Vademecum, 295, fig. 215. 
Il titolo del libro deve venire scritto sul bordo inferiore delle pagine. ${ }^{27}$ La scritta con l'elenco dei capitoli (tarğama) ${ }^{28}$ sia riportata nel giusto senso all'interno della coperta, sul lato dove si trova la basmala. ${ }^{29}$ L'utilità del titolo consiste nel permettere l'identificazione del libro e favorirne l'estrazione quando si trova collocato tra altre opere. Se il libro è posto a terra o su un supporto, la coperta dal lato della basmala e l'inizio dell'opera deve stare verso l'alto. Non si ecceda nell'inserire la ribalta all'interno del corpo del libro [come segnalibro], per non accelerarne la rottura, né si mettano libri di grande formato sopra altri più piccoli, con il rischio di farli cadere.

Non si riduca il libro a contenitore di fascicoli sciolti (karāriss), ${ }^{30} \mathrm{o}$ altro, né sia usato per cuscino, ventaglio, peso, appoggio, sostegno, e neppure per uccidere cimici o altri insetti [!]; non [si traccino] segni sulle pagine, sarebbe cosa grave! I margini o gli angoli dei fogli non devono venire piegati. Se serve un segnalibro, si faccia di carta e non di legno o materiali rigidi. Bisogna fare attenzione a non lasciare sulle pagine segni di unghia.

Sezione iv: [Come acquistare libri] ${ }^{31}$

Quando si prende in prestito un libro o si restituisce, è opportuno esaminarlo. ${ }^{32}$ Procedendo all'acquisto, si controlli con attenzione l'inizio, la fine, l'interno, l'ordine dei capitoli e dei fascicoli, si sfoglino le pagine, si valuti se c'è l'indicazione che il libro è stato oggetto di correzione. Se il tempo è limitato, prevalga il criterio della correttezza rispetto all'esame minuzioso; come disse al-Šăfi ${ }^{c} \overline{1}$, Dio sia soddisfatto di lui: 'Se vedo aggiunte e correzioni in un libro, sono sicuro della sua correttezza'. Un tale ha detto: 'Il libro non riluce se non è annerito', intendendo con ciò le correzioni (iṣlāh) che vi sono state apportate.

27 Cioè sul taglio di piede del libro.

28 Sembra che l'autore intenda con tarğama l'elenco dei capitoli, tuttavia nella frase che segue il significato non può che far riferimento al titolo. Il vocabolo ha infatti vari significati, tra $\mathrm{i}$ quali 'titolo di un libro, elenco dettagliato del contenuto, lista dei capitoli'. Si veda Gacek, The Arabic Manuscript Tradition, 17-18; Id., Arabic Manuscripts, 57-8. Nel trattato di al'Almawī, che segue lo schema di Ibn Ğamāca, viene precisato che '[l'elenco] dei titoli dei capitoli va posto all'interno della coperta opposta alla ribalta, affinché la scritta non venga a rovescio' ( $a l-M u^{c} \bar{\imath} d$ fi adab al-mufìd, 254).

29 In modo che, aprendo il libro, si trovi all'inizio.

30 Cfr. Gacek, Arabic Manuscripts, 210-13 (Quires).

31 Cfr. al- ${ }^{c}$ Almawī, al-Mu ${ }^{c} \bar{\imath} d$ fi adab al-mufìd, 254; Rosenthal, The Technique, 12.

32 Al- ${ }^{\mathrm{c}}$ Almawī aggiunge: 'al fine di verificare che non siano state dimenticate al suo interno delle note utili o altro' (al-Mu'i $\bar{\imath}$ fi adab al-mufid, 254). 
Sezione v: [Come copiare i libri] $]^{33}$

Quando si copia da libri di scienze sharic ${ }^{\mathrm{c}}$ itiche, è necessario che lo scriba si trovi in stato di purità rituale, sia rivolto verso la qibla, puro di corpo e di abiti, e scriva con inchiostro puro. ${ }^{34}$ Ogni scritto deve iniziare con la formula bismi llāhi l-rahmāni l-raḥim ('nel nome di Dio il Clemente il Misericordioso'). Se il libro inizia con una huṭba ('prefazione') che comprende l'espressione 'Lode a Dio l'Altissimo e preghiera al Suo profeta', si ponga appena dopo la basmala, a meno che già non si trovi in questa posizione. ${ }^{35}$

[Dopo la basmala e l'eventuale hutba] si copia il contenuto del libro. Alla conclusione di ciascuna parte $\left(\check{g} u z^{\circ}\right)$, ad esempio dopo la prima o la seconda, si scriva 'segue questo e quello', qualora la scrittura sia ancora incompleta. Quando invece è terminato, si scriva tamma l-kitāb... ('è finito il libro... [segue il titolo]'). In ciò c'è molta utilità.

Ogni volta che si scrive il nome di Dio, si facciano seguire eulogie che lo glorificano, quali tac ālā ('l'Altissimo'), subhāanahu ('Dio sia esaltato'), oppure 'azza wa-ğalla ('è potente ed eccelso'), taqaddasa ('sia santificato') o simili. Quando si scrive il nome del Profeta, si aggiunga al-șalāt calayhi wa-l-salām 'alayhi ('su di lui la preghiera e il saluto [di Dio]'), pronunciando al tempo stesso l'invocazione.

È consuetudine degli antichi e della generazione passata scrivere șallā llāhu calayhi wa sallama ('Dio preghi su di lui [il Profeta] e gli dia il saluto/la salute [eterna]'), questo forse in accordo con il versetto del nobile Corano 'Pregate anche voi su di lui e salutatelo di saluto di pace'. ${ }^{36} \mathrm{Ci}$ sono numerosi studi in proposito.

Anche se [la menzione del Profeta] ricorre ripetutamente, non si abbrevi la formula come fanno alcuni redattori disattenti, che scrivono $s l^{c}$, oppure s lm o s $l^{c} m$. Tutto ciò non si addice al Profeta, Dio preghi su di lui e gli dia il saluto. Numerose tradizioni attestano che la scrittura deve essere completa, evitando le abbreviazioni.

Se ricorre la menzione di un compagno [del Profeta], in particolare i più importanti, si aggiunga raḍiya llāhu canhu ('che Dio sia soddisfatto di lui'), ma non si scriva al-șalāt wa-l-salām ('la preghiera e il saluto') per nessuno eccetto i profeti, e immediatamente dopo [il loro nome]. Ogni volta che ricorre la menzione di un pio antenato (salaf) ci si comporti allo stesso modo o si scriva rahimahu lläh ('che Dio lo abbia in misericordia'), in particolare per i celebri imam e per le guide spirituali dell'Islam.

33 Cfr. al- ${ }^{\mathrm{c}}$ Almawī, op.cit., 254-6; Rosenthal, The Technique, 12-13.

34 Cfr. Gacek, Arabic manuscripts. 235-6 (Scribal etiquette).

35 Cfr. Gacek, Arabic Manuscripts, 200-3 (Prefaces of compositions).

36 Cor. 33:56. 
Sezione vi: [Sulla scrittura minuta] ${ }^{37}$

È opportuno evitare la scrittura minuta nella copiatura, poiché la calligrafia (hatt t) è segno: la più chiara è la migliore. Un nostro antenato quando vide una calligrafia minuta disse: 'Questa è la calligrafia di chi non è certo che Dio l'Altissimo gli darà una discendenza'. Un altro disse: 'Scrivi ciò che ti è utile per quando ne hai bisogno, e non quello che non ti sarà di nessuna utilità'. Alludeva al tempo della vecchiaia e alla vista indebolita. Talvolta dei viaggiatori si propongono di ridurre con una scrittura minuta il disagio nel trasportare [manoscritti pesanti]; il proposito è lecito, tuttavia l'utilità in ultima analisi è passeggera.

Usare inchiostro hibr è meglio che usare inchiostro midād, poiché assicura maggiore costanza nel tempo. ${ }^{38} \mathrm{Si}$ ritiene che il calamo ${ }^{39}$ non debba essere troppo rigido, tanto da impedire la scioltezza del movimento, ma neppure troppo morbido per non logorarsi in fretta. Un tale ha detto: 'Se vuoi migliorare la tua calligrafia lascia il pennino [cioè la punta del calamo] allungato, largo e con un taglio obliquo verso destra'. Il coltello per fare la punta al calamo e raschiare la carta deve essere molto affilato: non si usi se non a questo scopo. [La tavoletta] dove viene tagliata la punta al calamo deve essere molto solida. [Gli scribi] lodano in proposito la canna fârs $\bar{\imath}$ secca e l'ebano levigato.

Sezione vii: [Retto comportamento nella correzione del libro] ${ }^{40}$

Quando il libro è oggetto di revisione collazionandolo con l'originale corretto o [ricorrendo all'aiuto di] uno shaykh, è necessario introdurre la vocalizzazione e i punti diacritici, chiarendo i passi oscuri e verificando la correzione. Qualora sia necessario controllare ciò che si trova nel testo (matn) del libro nei confronti con ciò che è posto a margine, ed esplicitarlo, ci si scriva sopra un'annotazione. Lo stesso principio si estende alla nota a margine, con un'indicazione di preferenza; ad esempio se c'è nel testo il nome si riportano a margine le [singole] lettere, cioè $h$, seguita da $r$, quindi da $y$ e z; oppure [secondo una diversa lettura] con $\check{g}$ seguito da $y$

37 Cfr. al- ${ }^{c}$ Almawī, al-Muci $\bar{\imath}$, 256-7; Rosenthal, The Technique, 13.

$38 \mathrm{Hibr}$ è l'inchiostro composto da un elemento tanninico (noci di galla) e un sale metallico; midād da nerofumo e un legante. Si veda Gacek, The Arabic Manuscript Tradition, 27-8; Id., Arabic Manuscripts, 132-5 (Ink); Déroche, Manuel de codicologie, 120-4.

39 Sul calamo e gli altri strumenti di scrittura si veda Gacek, Arabic Manuscripts, 40-2 (Calamus), 294-6 (Writing implements and accessories); A. Grohmann, Arabische Paläographie, 1: 117-27 (Die Schreibgeräte); J. Sadan, Nouveaux documents sur scribes et copistes, 62-4 sull'epistola di Ibn Qutayba, al-Haț wa-l-qalam.

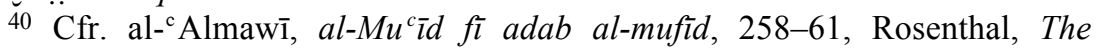
Technique, $14-15$. 
posta tra due $r^{41}$ È consuetudine indicare nella scrittura le lettere $m u^{c}$ ğama con i punti diacritici; per quelle muhmala ('sprovviste') ${ }^{42}$ c'è chi lascia come segno ( ${ }^{c}$ alàma) la loro omissione, e chi pone dei simboli, dei punti rovesciati o simili, o una piccola mezzaluna e altre indicazioni.

Dopo aver revisionato un testo, è opportuno segnalare con una piccola $h$ i luoghi che restano dubbi nel corso della consultazione e presentano possibilità [di interpretazione diverse], annotando sul testo o sulla copia: wa-huwa hața'... ('è un errore', = errata) in caratteri piccoli e riportando a margine: șawābuhu... ('giusto è...', = corrige), ${ }^{43}$ se si è ben certi di ciò.

[In caso di incertezza] si aggiunga una dabba ('chiavistello'), che ha la forma della testa di una $s$ (ص), da scriversi sopra la parola ma non unita ad essa. Se in una successiva revisione risulta che la parola è esatta, si aggiunga alla $s$ una $h$, che così diventa صح (șhhha, 'è giusto'), altrimenti si scriva la parola corretta a margine così come detto in precedenza. ${ }^{44}$

Se ricorre una parola spuria nel manoscritto, qualora si tratti di una singola parola ci si scrive sopra $l \bar{a}$ ('no') o si espunge; se sono più parole, una riga o più righe, volendo si può scrivere sopra la prima: min ('da [qui]...') oppure $l \bar{a}$ ('no'), e alla fine ilā ('fino a...'). Questo significa 'ometti da qui fino a lì'. Se è il caso, si espunga il tutto scrivendoci sopra con scrittura minuta, al fine di ottenere quanto ci si propone senza imbrattare la carta. Qualcuno pone al posto della scritta una fila di punti.

Se una parola viene ripetuta da parte dello scriba inavvertitamente, si espunga la seconda volta che ricorre, poiché la prima è giusta al suo posto. Questo purché essa non si trovi in fine al rigo: in tal caso è meglio cancellarla per un migliore inizio rigo, a meno che non sia annessa in uno stato costrutto.

Sezione viii: [Come si pone un'aggiunta allo scritto]

Se si vuole inserire (tahrīğg) ${ }^{45}$ qualcosa a margine, [processo] chiamato anche 'aggiunta' (lahaq), se ne contrassegni il luogo con una linea

41 L'editore riporta nel testo harizz, ma è da intendersi, in base a quanto segue, la parola priva dei diacritici, senza i quali può essere interpretata sia come harīz ('prezioso') che come ğarīr ('briglia'): sarà il contesto a far scegliere quale delle due letture è più sensata. Nella tradizione araba, il copista scrivendo una parola di incerta lettura preferisce non porre i diacritici sulla singola lettera, ma 'descriverla', ad esempio $ح$ muhmala (senza punti diacritici) o $m u^{c} \check{g} a m a$ (con punti diacritici).

42 Gacek, Arabic Manuscripts, 144-5 (Letter-pointing); 286 (Unpointed letters).

43 Gacek, Arabic Manuscripts, 234-5 (Scribal errors).

44 Gacek, Arabic Manuscripts, 170-1 (Omissions and insertions); 283-5 (Transcription marks).

45 Il vocabolo ha significati diversi, talvolta contrastanti, tra i quali 'edizione o redazione che comporta correzioni, selezione o riordinamento dei capitoli; omissione; estratto da un libro, citazione; inserimento' e altri (Cfr. Gacek, The 
leggermente curva fino all'inserto, meglio se verso destra. Poi si scriva l'aggiunta a partire dall'estremità del segno salendo verso l'alto della pagina, senza scendere in basso per l'eventualità che sia necessaria un'altra aggiunta. L'orientamento delle lettere è con la testa verso la destra [del foglio], ${ }^{46}$ sia che l'aggiunta si trovi sul margine destro che sinistro dello scritto. È opportuno tener conto della lacuna e dello spazio necessario [per integrarla] prima di scrivere. Se sono due righe o più si faccia in modo che la scrittura segua la fine della riga se l'aggiunta è sulla destra; l'inizio della riga [seguente] se è sulla sinistra. Non si continui a scrivere e tracciare righe a margine della pagina, ma si lasci uno spazio vuoto che renda possibile la cucitura. Inoltre si scriva alla fine dell'aggiunta șaḥha ('è corretto'); alcuni riprendono anche la parola finale della frase [precedente], quale segno di continuità del discorso.

\section{Sezione ix: [Annotazioni a margine $]^{47}$}

Non c'è impedimento nello scrivere glosse, notizie utili e avvertenze importanti ai margini di un libro di possesso. Non si annoti alla fine di quanto aggiunto șạha ('è corretto'), lasciando distinta in questo modo la vera aggiunta al testo. Alcuni precisano nello spazio sovrastante 'nota' o 'notizia utile' ( $f \bar{a}^{\jmath} i d a$ ), altri lo scrivono alla fine. Non si riportino se non informazioni importanti correlate al libro specifico, ad esempio un'avvertenza su questioni dubbie o su riserve, simboli o errori e simili.

Non si imbrattino le pagine aggiungendovi questioni o sezioni estranee, non si abbondi con le note tanto da nuocere [alla lettura] del libro o distrarre lo studente dagli argomenti trattati. Non si deve scrivere tra le righe; talvolta qualcuno lo fa tra righe distanziate con [inchiostro] rosso o altro colore, ma è meglio non farlo.

Sezione $\mathrm{x}$ : [Evidenziazione in rosso di capitoli e sezioni] ${ }^{48}$

Non c'è obiezione allo scrivere con inchiostro rosso i nomi di capitoli, rubriche e sezioni: ciò rende più evidente l'articolazione delle parti del discorso. ${ }^{49}$ Allo stesso modo, non c'è obiezione [nello scrivere in rosso] nomi, scuole, detti, metodi, generi, vocaboli, numeri o simili. Quando si opera così si esplicitino i relativi termini nell'introduzione del libro, al fine di farne capire il significato a chi si accinge [alla lettura]. Contrassegnano in rosso molti tradizionisti, giuristi, studiosi di diritto musulmano, volendo abbreviare. Se non si procede come menzionato in merito all'evidenziazione in rosso di capitoli, sezioni e rubriche, li si distingua con un tratto grosso del calamo e l'estensione della forma della lettera (mašq) lungo la riga, o

\footnotetext{
Arabic Manuscript Tradition, 39).

46 Procedendo la scrittura araba da destra a sinistra, si scrive dal basso in alto.

47 Cfr. al- ${ }^{\mathrm{c}}$ Almawi $, a l-M u^{c} \bar{\imath} d, 265-6$; Rosenthal, The Technique, 17.

48 Cfr. al- ${ }^{\mathrm{c}}$ Almawī, al-Muci $\bar{\imath} d, 266$; Rosenthal, The Technique, 18.

49 Cfr. Gacek, Arabic Manuscripts, 227-9 (Rubrics and rubrication),
} 
con simili procedimenti di scrittura, questo al fine di facilitare la suddivisione voluta.

È consigliabile separare due argomenti con un cerchio, una rubrica o un tratto di penna grosso, in modo che non ci sia continuità di scrittura [tra quanto precede e quanto segue], poiché la difficoltà di dedurne il contenuto comporta una perdita di tempo. Solo gli ignoranti non procedono in questo modo.

Sezione xi: [La cancellatura $]^{50}$

Cancellare (darb, tracciando una riga sopra la parola $)^{51}$ è meglio che raschiare $(h a k k)$, in particolare nei libri di tradizioni del Profeta, poiché altrimenti ciò che era scritto non è più conoscibile o può essere solo oggetto di congettura, e inoltre perché una scrittura conservatasi a lungo ora va perduta. L'atto [di raschiare] è più grave: talvolta provoca la foratura della carta, un danno maggiore, e il suo indebolimento. Dal momento che rimuove punti diacritici e vocalizzazione, la cancellazione è maggiormente consigliabile.

Se il libro viene corretto [con l'aiuto di] uno šayh o tramite la collazione, si segni il luogo dove [la consultazione] o la collazione giungono nella revisione, o quant'altro risulti utile. Qualora questo avvenga in relazione all'audizione delle tradizioni del Profeta ( $\operatorname{sam}^{c}$ al-hadit $\underline{\text { }}$ ), si contrassegni il passo cui si è giunti nel primo, secondo e successivi incontri, fino alla fine. Al-Hațị [al-Bag̀dādī $]^{52}$ disse che, se si deve emendare qualcosa, si faccia la correzione con una scheggia di tek o di altro legno duro, evitando di immergerlo (al-šarīb) ${ }^{53}$

\section{3) Ibn al-Hāăğğ}

Nella sua biografia degli uomini illustri dell'VIII secolo dell'egira, Ibn Ḥağar al- ${ }^{c}$ Askalānī (m. 852/1449) riporta una breve nota su Ibn alḤāğğ. ${ }^{54}$ Giurista di scuola malikita, egli si recò dal nativo Maghreb in

50 Cfr. al- ${ }^{\mathrm{c}}$ Almawī, $a l-M u^{c} \bar{\imath} d, 261-2$; Rosenthal, The Technique, 15-16.

51 Cfr. Gacek, Arabic Manuscripts, 48 (Cancellations/deletions).

52 L'autore (m. 473/1071) è noto, oltre che per la sua storia di Baghdad e importanti opere biografiche e sul hadīt, per il trattato di etica dal titolo al$\breve{G} \bar{a} m i^{c}$ li-ahlāq al-rāwī wa-ādāb al-sāmi $i^{c}$ (Bayrūt, 1966), accostabile per certi aspetti all'opera di Ibn al-Ğamăc $\bar{a}^{\mathrm{c}}$.

53 Il passo con cui si conclude il capitolo risulta confuso e slegato dal contesto, anche perché nel ms. figura al-tarīb ('rimprovero'?), emendato dall'editore in al-šarīb. (172, nota 4).

54 Ibn Hağar, al-Durar al-kāmina, 4: 355-6, $\mathrm{n}^{\circ} 4490$ (al-Fārisī è da correggere in al-Fāsī); ripreso da al-Ḥăğğì Halīfa, Kašf al-z̧unūn, 5: 474, n 11.690. Notizie su Ibn al-Hāğğg sono riportate anche nelle opere di al-Șafadī, alWāfl̃ bi-l-wafāyāt, 1: $237 \mathrm{n}^{\circ} 157$ (il suo libro è chiamato Kitāb al-Bidac); Ibn Farhūun, al-Dībāğ al-mudahhab, 327-8; al-Suyūṭi, Husn al-muḥādara, 1: 459, nº 
Egitto e da qui effettuò il pellegrinaggio alla Mecca. Si legò allo shaykh Abū Muhammad b. Ab̄̄ Ğamra e ne raccolse l'insegnamento, distinguendosi per integrità e ascetismo. 'Scrisse al-Madhal_-annota Ibn Hağar - un'opera che contiene molte osservazioni utili, nella quale mette in luce i vizi e le innovazioni nell'operato della gente che agisce con negligenza: la maggior parte di queste azioni è da condannare, ma alcune sono tollerabili'. Morì nel 737/1336.

Tra le opere di Ibn al-Hāăğğ figura al-Madhal ilā tatimmat al-a $a^{c} m \bar{a} l b i-$ tahsīn al-niyyāt wa-l-tanbīh ${ }^{c} a l \bar{a} b a^{c} d$ al-bida ${ }^{c 55}$ spesso citato in forma abbreviata al-Madhal (talvolta al-Mudhal), trattato contro le 'innovazioni' di carattere morale, giuridico e religioso. ${ }^{56}$

Esponendo i motivi della redazione dell'opera, Ibn al-Ḥăğğ rende omaggio al suo maestro, Ibn Abī Ğamra. Rifacendosi al pensiero di alǴazzālî, egli insiste sul principio che l'atto di culto è soddisfatto solo se è preceduto dalla niyya, quale espressione del cuore e parte preminente dell'atto stesso. ${ }^{57}$ La preoccupazione maggiore, presente in ogni pagina del Madhal, nasce dal timore che prendano radice in tutte le classi sociali comportamenti contrari alla tradizione. ${ }^{58}$ In merito alle attività artigianali, le direttive di carattere etico-religioso di Ibn al-Hāăğğ 59 si accostano per numerosi aspetti a quanto prescritto nei manuali di hisba (ufficio di vigilanza sui mercati e sulla morale pubblica) sui compiti del muhtasib

76; al-Š́a ${ }^{c}$ rānī, al-Tabaqāt al-kubrā, 1: 273.

55 Cfr. GAL 2: 95; S 2: 95: Mudhal al-šar ${ }^{c}$ al-šarīf / Mudhal ilā tanmiyat alamal... Mi sono servito della ristampa dell'edizione egiziana del 1929.

56 Ms., cfr. GAL 1: 482; $S$ 1: 883.

57 Al-Madhal, 1: 12. Secondo al-Ġazzālī, 'Tutti sono destinati a perire eccetto quelli che conseguono la 'scienza'; anche questi periscono eccetto quelli che operano; anche questi periscono se non agiscono con cuore sincero: l'opera ( $\left.{ }^{c} a m a l\right)$ senza niyya è mero sforzo físico, la niyya senza sincerità è ipocrisia' (Ihy $\left.\bar{a}^{\supset}, 4: 351\right)$.

58 Non tutte le innovazioni sono proibite. Riprendendo la classificazione giuridica tradizionale, Ibn al-Hāăğğ distingue varie categorie di $\operatorname{bid}^{c} a$, spaziando da quelle lecite a quelle vietate, e porta degli esempi significativi (il primo è in certa misura pertinente agli artigiani del libro): $b_{i d}^{c} a$ wāğiba, 'obbligatoria', es. i libri di scienza (religiosa), anche se non sono opera degli uomini del passato, poiché l'cilm era nei loro cuori; o i diacritici nella scrittura del Corano; mustahabba, 'raccomandabile'; mubāha, 'lecita'; makrūha, 'riprovevole'; muharrama, 'vietata' (al-Madhal, 2: 257). Sull'evoluzione del concetto di bid'a, si veda in particolare Rispler, 'Toward a New Understanding', e fonti citate.

59 Anche Ritter ha sottolineato che opere quali il Madhal si inseriscono nella letteratura di carattere etico-religioso più che giuridico-istituzionale ('Ein arabisches Handbuch', 25). 
incaricato di verificare pesi, misure, qualità e prezzi delle merci, e inoltre 'ordinare il bene e vietare il male'. ${ }^{60}$ Nei manuali esaminati non ho trovato tuttavia cenno ai copisti, ai librai e ai rilegatori, verosimilmente perché il loro non è ritenuto un 'mestiere' assimilabile alle comuni attività di mercato, legato com'è a valori morali e religiosi, e il prodotto del loro lavoro è ben diverso da una comune merce. ${ }^{61}$

L'ultima parte del trattato di Ibn al-Hāğğg̀ è riservata all'etica del lavoro. Tra le categorie di persone destinatarie dei suoi ammonimenti figurano chi produce e chi vende la carta, il copista, il legatore, il decoratore di libri.

Ibn al-Ḥăğğ non manca di sottolineare il legame tra i mestieri di cartaio, copista e rilegatore con la religione. ${ }^{62}$ Il Corano-osserva l'autore-come pure gli altri libri religiosi sono scritti su carta, ${ }^{63}$ il che nobilita tale supporto e chi lo produce. Il copista ha un compito ancora più nobile, poiché con la sua opera diffonde la parola di Dio, associando scrittura, recitazione e riflessione. Nel copiare il Corano deve seguire la vulgata di ${ }^{~} U$ țān, anche in quei punti che suscitano perplessità per una grafia inconsueta. Della massima importanza è la scansione delle lettere, poiché 'la calligrafia migliore è quella più leggibile'. Il copista non deve prestarsi a scrivere parole in lingua non araba, di cui non conosce il significato. L'artigiano che rilega i libri deve padroneggiare l'arte del mestiere, non sbagliando l'ordine dei fascicoli, non confondendo volumi

60 Cfr. Cook, Commanding Right. Sulle opere di hisba si veda Shatzmiller, Labour, 71-sqq., e inoltre Ghabin, Hisba; Buckley, 'The Muhtasib'; Vercellin, 'Hisba'; Raymond e Wiet, Les Marchés, 36-sqq. (considerazioni di Ibn al-Hāğğğ 39, 72, 78-80); la Nihāyat al-rutba di al-Šayzarī; i Ma 'ālim al-qurba, di Ibn alUhuwwa, autore egiziano contemporaneo di Ibn al-Ḥāğğ. La figura e i compiti del muhtasib e del suo agente ( ${ }^{c}$ arîf) sono analizzati negli studi sulla vita sociale nella città islamica medievale; si veda sopra, nota 6 .

61 Mi sembra significativo che il recente dettagliato studio di A. Ghabin citi a questo proposito solo Ibn al-Hāăğğ, non trovando negli altri trattati alcun riferimento alle arti del libro (Hisba, 147).

62 Le sue accorate raccomandazioni fanno pensare che i reali comportamenti degli artigiani fossero alquanto diversificati. In base alle fonti esaminate da alHazīmī, nell'VIII secolo dell'egira furono distrutte le opere dell'ebreo convertito Faḍl Allāh b. Ubayy; una copia del Fuṣūṣ al-hikam di Ibn 'Arabī; i libri di Muhammad b. al-Hațîb; da parte sua, Ibn Taymiyya acquistò i trattati alchemici di Ibn al-Ğābī al-Ḩațīb e li 'lavò' per cancellarne lo scritto (alHazīmī, Haraq al-kutub, 60-4).

63 Evidentemente l'uso della pergamena era ormai molto limitato al tempo di Ibn al-Hāğğ. 
e rispettivi proprietari, onorando i tempi di consegna. Nella decorazione della coperta non è lecito usare oro o argento. È opportuno che il rilegatore rifiuti di operare su libri quali la Torah, il Vangelo, i Salmi, contenenti scritture celesti contraffatte. Da parte sua, il committente è tenuto ad accordarsi preventivamente con il rilegatore su materiali e costi.

Anche il nostro autore, come altri giuristi del tempo, non manca di condannare cartai, copisti e rilegatori che con la loro opera favorivano la diffusione delle 'falsità' $(k i \underline{d} b)$ contenute nei popolari romanzi di cavalleria (siyar), oggetto di recitazione pubblica da parte dei cantastorie, come la storia di al-Battāal e quella di ${ }^{c}$ Antara, che distoglievano i fedeli dai sermoni dei pii predicatori. ${ }^{64}$

\section{Dal Kitāb al-Madhal: 65}

I. Proposito del cartaio (warrāq $)^{66}$

Sappi-che Dio ci assista — che questo [la carta] è uno dei mezzi più importanti per accostarci al Signore, qualora il proposito sia buono. Infatti il nobile Corano è scritto su carta, così il suo commentario (tafsīr), 'l'abrogante e l'abrogato' e le scienze correlate. Lo stesso vale per il hadìt del Profeta, il suo commento (rarhh) e ciò che contiene di massime, significati, utili insegnamenti, di numero incalcolabile; e ancora per i libri di giurisprudenza e delle diverse scienze sharicitiche, per gli scritti sull'elemosina, sui contratti di vendita, di affitto, di procura, e molto altro di cui l'uomo necessita. Tutto questo riveste molta importanza per la religione (...).

A ciò si aggiunge il proposito della fede e della ricompensa [divina]; talvolta però si opera al contrario, ad esempio vendendo carta a chi si può presumere ne farà usi illeciti o sconvenienti. Non sono leciti [libri che descrivono] fatti iniqui e simili, e neppure menzogne come la storia (qișsa) di al-Batțāl o di ' Antara; ma l'elenco sarebbe lungo. Quanto a quelli sconvenienti, sono le storielle scherzose di cui la gente si diletta. Bisogna guardarsi da tutto ciò per non rientrare nelle parole dell'Altissimo: ' $\mathrm{O}$ voi

64 Rimando in proposito al saggio 'Critical Attitudes'.

65 Su cartai, copisti e rilegatori si vedano i rispettivi capitoli nel Manuel de codicologie arabe di Déroche; per la comprensione dei termini tecnici è stato molto utile il glossario di Gacek, The Arabic Manuscript Tradition e Supplement; sulla produzione di carta nel mondo islamico, si veda Bloom, Paper before Print.

66 Al-Madhal, 4: 79-83. Dato lo scopo prevalentemente tecnico della traduzione, vengono omessi alcuni passi in cui l'autore si dilunga in considerazioni di ordine morale, biasimando venditori e artigiani poco coscienziosi e poco rispettosi della $\check{s} a r \bar{l}^{c} a$, in un 'tempo caratterizzato da molte discordie e sedizioni'. 
che credete! Perché dite ciò che non fate? È grandemente ripugnante presso Dio che voi diciate quello che non fate' (Cor. 61:2-3). Questo perché se [il cartaio] vende carta a chi poi vi scrive le cose menzionate, compie un'azione che non ha manifestato con le labbra né si è proposto con il cuore. Se il venditore afferma, come avviene nella maggior parte dei casi, di non conoscere la condizione del compratore, gli si può ribadire che è suo dovere condurre i musulmani sulla via della purezza e dell'integrità. È comunque raro di questi tempi non avere conoscenza dello stato degli acquirenti: nella maggior parte predomina l'ignoranza (...).

È opportuno che si guardi dalla truffa, tentando di vendere per quattro dirham una risma (dast) di carta che ne vale tre. Il prezzo della carta è diverso a seconda della qualità: può essere di bianchezza (bayāọ) e levigatezza $(s i q \bar{a} l)$ superiore; può essere fabbricata in estate $\mathrm{o}$, tendendo al bruno (samra) e mancando di levigatezza o bianchezza, essere prodotta in inverno. Se è così, [il cartaio] deve indicarlo con chiarezza per evitare l'inganno; qualora non lo facesse, rientra per la sua dissimulazione in quanto disse il Profeta: 'Colui che ci imbroglia non è dei nostri' ${ }^{67} \mathrm{La}$ vendita all'acquirente non deve aver luogo priva di contrattazione (musāwama) o a premio fisso (murābaha). Se avviene per contrattazione, questo è il modo migliore e più sicuro; se invece è a premio fisso, deve essere soddisfatta la condizione menzionata a proposito del mercante di stoffe. Tutto ciò che è stato ricordato in merito al mancato esame da parte dell'acquirente, quando egli si reca al mercato o si ferma presso qualche venditore, vale per tutti gli operatori.

È opportuno fare attenzione, nell'acquistare carta, che non sia il momento in cui i lavoratori che la producono hanno scoperte le parti intime, poiché essi in genere si pongono ai lombi una stoffa succinta che si inzuppa di acqua lasciando le cosce scoperte. Se [1'acquirente] entra [nella cartiera] e si trova in questa situazione, compie un atto che invalida l'essenza stessa della sua niyya.

[Il cartaio] deve aver cura nel non mescolare carta leggera a carta di buona qualità adatta alla copiatura, poiché questo è un imbroglio nei confronti del compratore, dal momento che la carta leggera non sopporta la raschiatura (kaštt) per il suo spessore ridotto. Se egli sa che l'acquirente è un copista, deve dargli ciò che gli conviene; se sa che è uno che scrive lettere e simili, è lecito vendergli carta leggera, ma solo dopo averlo informato in merito.

Non si faccia alcun uso di carta che presenta una scritta se non dopo averne conosciuto il contenuto: ci possono essere versetti coranici o hadīt del Profeta, o uno dei nomi dell'Altissimo, oppure il nome di un profeta o di un angelo. Tutto ciò è vietato per rispetto della legge, poiché alcuni potrebbero calpestare queste scritte, o compiere simili atti, il che sarebbe il peggiore degli abomini. Dio ce ne guardi! [Il cartaio] non deve permettere

${ }^{67}$ Cfr. Muslim, Șahīḥ, Īmān, 164 etc. 
a nessuno degli operai comportarsi in modo disonorevole; se qualcuno agisce così, sia allontanato dal lavoro e un altro prenda il suo posto, purché rispetti le condizioni menzionate, compiendo la preghiera nei tempi stabiliti. (...)

II. Proposito del copista $(n \bar{a} s i h)^{68}$

Sappi che il copista merita maggiore compenso e remunerazione [celeste] del cartaio, poiché opera con grande devozione se copia di continuo il Libro di Dio, oppure il hadìt del Profeta, opere di giurisprudenza o di altre scienze shari itiche. Per quanto riguarda il Corano, il copista mette così assieme recitazione $($ tilāwa - la pietà più autentica-e scrittura, specialmente se scrive con metodo e nel contempo riflette sui significati delle parole. Se scrive hadī $\underline{t}$ del Profeta è a lui vicino nella remunerazione; tra i meriti che ne derivano, viene ricordato che 'gli angeli continuano a pregare per colui che scrive l'eulogia per il Profeta per tutto il tempo che essa perdura in quello scritto'. Il copista deve guardarsi dal copiare cose diverse dalle scienze shari itiche, poiché se facesse ciò verrebbe meno il suo proposito, sul quale si era impegnato. (...)

[Il copista] deve inoltre guardarsi dal copiare quanto ricordato di opere menzognere, come la storia di al-Batțāl e di ${ }^{\mathrm{c}}$ Antara e simili-questo è vietato, come pure storielle scherzose-il che non è desiderabile. Egualmente non copi per l'iniquo o per chi propende per l'ingiustizia o consegue guadagni sospetti, per non ricadere nelle parole dell'Altissimo: ' $O$ voi che credete! Perché dite ciò che non fate...?' (Cor. 61:2-3).

Il copista è tenuto a ben distinguere le lettere nel suo scritto, senza introdurre legamenti [inconsueti] nella sua calligrafia, tanto che questa possa essere compresa solo da chi ne ha profonda conoscenza: le lettere devono essere chiare e ben distinte. Non deve inoltre omettere nessun punto [diacritico] nelle lettere che ne necessitano, poiché + è diversa da $ت$ e ث,

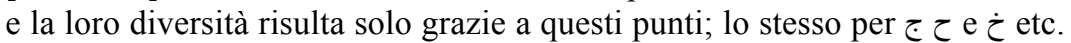
Riponga ogni cura in ciò, poiché così facendo ne trae utilità gran numero di musulmani. Al contrario agiscono ai giorni nostri molti compilatori di documenti, dal momento che inseriscono abbreviazioni che solo loro sono in grado di comprendere; qualcuno addirittura non sa leggere quanto scritto da altri scrivani, poiché ciascuno ha i propri metodi ed è difficile che un altro capisca. Tutto ciò è contrario alla Sunna. Si racconta che il Profeta disse a $\mathrm{Mu}^{c}$ āwiya: 'Metti [cotone] nel calamaio, taglia obliquamente la punta del calamo. [Quando scrivi la basmala], traccia diritta la + , separa

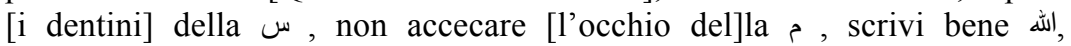
estendi الرحمن , da' bella forma a الرحيم . Poni il calamo dietro l'orecchio, ti farà ricordare chi detta' ${ }^{69}$ Nell'eventualità che lo scrivano muoia, o sia difficoltoso rintracciarlo, vanno persi i diritti dei musulmani e i loro

68 Al-Madhal, 4: 83-7.

69 Cfr. Rosenthal, The Technique, 13, da al- ${ }^{\mathrm{c}}$ Almawī. 
contratti di matrimonio scritti nella forma menzionata, dal momento che nessun altro è più in grado di leggere quei documenti.

[Il copista] non deve scrivere con un inchiostro (hibr) che corrode la carta: in ciò vi è perdita di beni e di scienza, in modo particolare se si tratta della copia di un libro perduto o prezioso; lo stesso se si usa inchiostro che si cancella rapidamente dal foglio. Quanto allo scrivere con un inchiostro $(\text { mida } d)^{70}$ che annerisce la carta tanto che le lettere rimangono confusefenomeno ben comune - deve senza alcun dubbio venire vietato, a meno che lo scriba non verghi una lettera [copiandola] da un documento a un altro e simili operazioni; purché non vi si trattino sentenze giuridiche, com'è il caso del libro del Qāạ̄ ['Iyāụ], dovendo allora sottostare alle condizioni menzionate a proposito di figh, procure e simili. Si dice che 'la calligrafia migliore è quella più leggibile'. Conviene che lo scriba, quando si accinge alla sua opera, abbia compiuto le abluzioni rituali, almeno all'inizio della seduta. Se però sta copiando il Libro di Dio, le abluzioni sono necessarie ogni qualvolta sopravvenga uno stato di impurità (hadat ).

Che [il copista] si astenga dal tirare per le lunghe il lavoro, ma rispetti la parola data, poiché questo rientra in una genuina pratica devota. Non la macchi con il venir meno a quanto stabilito, dicendo 'domani o dopodomani', senza poi eseguire il lavoro. Si guardi dal perdere la fiducia [dei suoi committenti]. Non deve inoltre agire come alcuni fanno, cioè assumere da varie persone l'incarico di copiare e poi farlo solo per questo e per quello, senza che [gli altri] ne siano a conoscenza. Ciò incrina la loro fiducia, poiché [tale atteggiamento è indice di] altezzosità e cupidigia combinate assieme.

È doveroso che lo scriba non svolga la sua opera nella moschea, anche se vi si trova per atto di culto: ciò potrebbe, per una causa o un'altra, contaminarla. Se sente l'appello alla preghiera, egli deve lasciare il lavoro che sta effettuando, porgere attenzione alle parole del $m u^{\circ} a \underline{d} \underline{d}$ din e prepararsi per non mancare alla șalät con gli altri fedeli nel tempo stabilito. O Dio, che l'ad̄àn non avvenga mentre lo scriba è [concentrato] nello scrivere sul foglio, [teme] di alterare il suo tratto se si interrompe e così indugia fino al completamento dello scritto... Lo stesso se sta tracciando righe sulla carta e non vuole sollevare la mano prima di averle finite. Ciò non è tuttavia da biasimarsi, poiché rientra nella buona pratica di lavoro ed è di esortazione ai suoi fratelli musulmani. Ma Dio ne sa di più.

Lo scriba non deve tener conto di quanto alcuni gli dicono ai giorni nostri, e cioè di scrivere una copia completa del Corano (hatma ${ }^{71}$ basandosi su una scrittura che diverge dal mușhaf su cui concorda la Umma, in base a quanto essa ha ricevuto per mano di ${ }^{\mathrm{c}} \mathrm{Utmān} b .{ }^{\mathrm{c}} \mathrm{Affān}{ }^{72}-$ Dio lo

${ }^{70} \mathrm{Si}$ veda nota 38 .

${ }^{71}$ Cfr. Gacek, Arabic Manuscripts, s.v.

${ }^{72}$ Le principali peculiarità ortografiche del cosiddetto Corano di ${ }^{\mathrm{c}} \mathrm{Ut}$ tmān sono analizzate da Bergsträsser e Pretzl in $G d Q$ 3: 26-53. 
abbia in misericordia. Disse Mālik che il Corano è scritto nel Libro Primo [l'archetipo celeste]. Nulla è lecito diverso da questo, né l'inclinare verso la pretesa di chi se ne discosta, sostenendo che tanto il volgo non conosce lo scritto (marsüm) coranico, facendo errare la gente nella recitazione. (...) Quelli del volgo che non conoscono la scrittura coranica non devono leggere se non dopo il suo apprendimento, altrimenti introdurranno errori nel testo condiviso dalla Umma. (...)

Lo scriba deve guardarsi dallo scrivere la hatma nella lingua degli ${ }^{\mathrm{C}}$ Ağam, ${ }^{73}$ poiché Dio l'Altissimo ha fatto scendere il Corano in 'lingua araba chiara' ${ }^{74}$ e non nella loro lingua. Mālik - che Dio lo abbia in misericordia-biasimava la scrittura del Corano in parti disgiunte, osservando che Dio ha detto 'a noi sta raccoglierlo'; ${ }^{75}$ ma alcuni, nonostante ciò, lo suddividono. Se egli detestava la suddivisione in $a \grave{g} z \bar{a}^{2}$, figuriamoci come avrebbe giudicato chi altera la lingua araba chiara! Questo purtroppo avviene nel nostro tempo, tanto che alcuni adattano la lettura/recitazione del Corano alla 'ağamiyya e così scrivono il testo. $\mathrm{Si}$ giunge a mettere assieme in un unico scritto parti in arabo e parti in ${ }^{c} a \check{g} a m \bar{l}$, scrivendo due-tre versetti in arabo per poi riportarli in ${ }^{c}$ ağamī. Tutto ciò contrasta con quanto condiviso unanimemente dalla prima generazione musulmana, dai pii antenati e dagli ulema, Dio li abbia in misericordia.

III. Proposito dell'artigiano che rilega Corani e altri libri ${ }^{76}$

Sappi che questo è uno dei più importanti lavori per la religione perché permette di proteggere i Corani, i libri di hadīt e di scienze sharicitiche. È necessario in tutto ciò il proposito precedentemente menzionato in relazione al copista, poiché [l'opera del rilegatore] è di ausilio alla salvaguardia del frutto della sua fatica, oltre alla bellezza che dà al libro stesso e la dignità che gli conferisce. Quando l'artigiano esce di casa, assume quanto gli compete dei propositi del dotto e dell'istruito, quindi esprime l'intenzione di aiutare i suoi fratelli musulmani con il suo mestiere, dedicandosi alla cura dei loro Corani e dei loro libri; infine manifesta la niyya della fede e della ricompensa [celeste]. Se qualcuno dice che a questo - o altri artigiani - non si addice la niyya del dotto poiché quest'ultimo si reca alla moschea ad apprendere e insegnare in ottemperanza al proprio proposito, mentre per lui non può essere così poiché è immerso nella propria attività, la risposta è che non esiste differenza tra il dotto e l'artigiano, dal momento che sia lui che tutti coloro che svolgono simili lavori devono conoscere quattro 'scienze': 1. l'arte del mestiere ('ilm $\left.a l-\sin \bar{a}^{c} a\right)$; 2. il lessico tecnico ad esso riferito; 3. la coscienza di se stessi, che

73 bi-lisān al- cağam, 'nella lingua dei Persiani'. Lascio tuttavia il termine in trascrizione, poiché verosimilmente l'A. intende qualsiasi lingua non araba.

74 Cfr. Cor. 16:103 'Ma la lingua di quello cui pensano è barbara ( $\left.{ }^{c} a g ̆ a m \bar{\imath}\right)$, mentre questo è arabo chiaro'; 26:165.

75 Cor. $75: 17$.

76 Al-Madhal, 4: 87-92 
ognuno dovrebbe avere, per ciò che concerne le azioni di culto, i doveri etc.; 4. la prudenza necessaria da parte di colui che riceve un incarico nei confronti del committente. (...)

È auspicabile che, quando [l'artigiano] si reca alla sua bottega, si comporti in conformità con la Sunna, come già ricordato in merito all'entrare e uscire da casa: faccia precedere la destra alla sinistra pronunciando la tasmiya ${ }^{77}$ e quanto riferito dalla tradizione. Effettui due $r a k^{c} a$, poiché la preghiera è un legame tra l'uomo e il Signore, quindi si metta all'opera. Se nella bottega non c'è un luogo adatto alla preghiera, la sostituisca con la menzione di Dio. (...) Si astenga dagli imbrogli che lo adombrano nel suo mestiere, poiché, come ha detto il Profeta, 'la fede è comportamento leale' ${ }^{78}$ (...)

[Il committente] si guardi dal comportamento di alcuni: deve consegnare il libro al rilegatore a condizioni chiare e non generiche. Così evita che [il rilegatore] faccia tutt'uno di pelle, fogli di sguardia (bițāna), seta e suo compenso. [Sono da tenere in considerazione tre possibilità:] 1. [Il committente] fornisca da parte sua al rilegatore pelle, sguardie e seta e gli chieda di lavorare con questo materiale; 2. [oppure] il rilegatore indichi con precisione tutto ciò che occorre e il suo costo; 3 . [il committente] lo incarichi di acquistare i materiali necessari, se già non li possiede, quindi si stabilisca il compenso per il lavoro. Questi tre punti sono di facile applicazione ed evitano noie. (...) Il proprietario del libro si sentirà responsabile del valore di pelle, sguardie e seta [fornite] e del giusto compenso al rilegatore; quest'ultimo si sentirà responsabile di ciò che riceve dal proprietario. C'è da stupirsi come alcuni rilegatori ricevano [ordini di] lavoro su libri di scienza e si comportino in modo non lecito.

[Il rilegatore] deve fare bene attenzione alla carta che usa per le sguardie. Prevale presso alcuni artigiani del nostro tempo l'uso di carta senza curarsi di cosa vi sia [scritto]: ciò non è lecito. Vi può figurare il nobile Corano o un hadīt del Profeta o nomi di angeli o di profeti - su di loro il saluto. Se è così non è lecito impiegare questa carta né abusarne per il rispetto che merita e il suo valore. Se invece vi sono nomi di ulema e di pii antenati-Dio sia soddisfatto di loro-o passi di scienze sharic ${ }^{\mathrm{c}}$ itiche, l'uso è sconsigliabile, anche se non si giunge al grado di divieto come nel caso sopra menzionato. Colui che cerca la scienza a maggior ragione si astenga dal compiere azioni riprovevoli. Se l'artigiano sa o pensa di fare qualcosa che rientra in tutto ciò, non lo faccia! Lavori solo dopo che gli è stata chiarita la relativa prescrizione e ne abbia preso atto.

Non c'è inconveniente nel ricoprire internamente la pelle [della coperta] con carte contenenti cifre; non è biasimevole, ma il rilegatore deve essere prudente e accertarsi che non facciano parte di un quaderno (daftar) perduto

77 La formula bism ${ }^{i} l l a ̄ h i$.

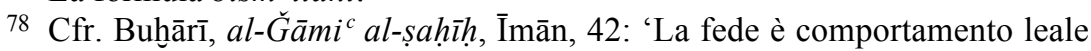
verso Dio, il Profeta, il Suo Libro, gli imām dei musulmani e la loro comunità'. 
da qualcuno, di cui abbisogna per non subire perdite di denaro. Se agisce in questo modo, l'artigiano contribuisce a salvaguardare i beni della gente. Deve inoltre fare attenzione al numero dei fascicoli del libro e dei suoi fogli, senza anticiparne o posticiparne la giusta posizione. Ciò rientra tra le raccomandazioni che mirano a tenere lontano l'imbroglio. L'artigiano ha bisogno di conoscere i richiami [a fondo pagina, istihrāăg] per unire coerentemente il testo con ciò che segue [nel foglio successivo], oppure deve essere reso partecipe [dallo scriba o dal proprietario del libro] nel conoscere [il giusto ordine dei fogli]. Oltre a tutto ciò, deve essere prudente nell'affidare il lavoro ad altri artigiani o garzoni incompetenti, affinché non si confonda libro e suo proprietario, cosa che spesso si verifica nel nostro tempo. [Il rilegatore] pena nel suo lavoro, ma talvolta con la fatica consegue guadagni illeciti se pretende dal proprietario [del libro più del dovuto]. Se questo avviene, l'artigiano deve restituire [il maltolto], anche se in più momenti, per rimettersi sulla buona strada, tenendo per sé solo il compenso inizialmente [pattuito] (...).

L'artigiano non deve rilegare nessun libro appartenente a gente che professa religioni false, poiché così facendo favorisce la loro miscredenza: chi aiuta qualcuno in un'azione ne condivide [la responsabilità]. Un secondo aspetto simile o a questo vicino, è che queste persone sono gratificate nella loro religione, poiché se vedono un musulmano che le aiuta si convincono di essere nel giusto. Qualora [il rilegatore] sappia che il libro datogli è tra quelli rivelati-come la Torah, il Vangelo, i Salmi-deve tenere conto della prescrizione che vieta [la sua opera]; questo perché è certo che essi [ebrei e cristiani] vi hanno apportato cambiamenti e contraffazioni. Non conoscendone i luoghi [dove ciò è avvenuto], meglio tralasciare tutti questi libri. Se gli viene data un'opera scritta in siriaco o in ebraico, e simili, non rileghi nulla di tutto ciò! Mālik—Dio ne abbia misericordia - disse a proposito delle formule magiche $(r u q \bar{a})$ in lingua non araba: 'Come fai a sapere [cosa significano]? Forse è miscredenza'. Bisogna stare attenti ad evitare tutto ciò che di simile viene escogitato nel cuore dell'uomo.

È opportuno che colui che cerca la scienza o altri che ricorrono all'opera del rilegatore si cautelino nei confronti degli artigiani che agiscono in questo modo e se ne astengano dopo averli istruiti sul retto comportamento: forse si pentiranno o si ravvederanno. Questo se era impossibile [al committente] affidare il lavoro a chi ne ha i requisiti, come menzionato a proposito della disapprovazione dell'azione riprovevole. (...) Cosa pensare dell'artigiano che rilega [libri agli iniqui] e attraverso la sua opera permette loro di conservare ciò che è proibito dalla nobile legge?

Che [il rilegatore] non decori una coperta servendosi di un calamaio che contiene oro o argento, poiché non è lecito usarli, come pure non è lecito servirsi di queste sostanze nel processo di rilegatura. Inoltre non deve rilegare per gli iniqui. Ciò per due motivi: 1 . chi aiuta qualcuno diventa corresponsabile, come abbiamo ricordato; 2. la maggior parte della loro 
ricchezza è illecita, e va a finire che l'artigiano si sforza nel suo lavoro per ricavarne un guadagno lecito ma invece lo consegue illecitamente. Si guardi da tutto ciò! Prevale tuttavia il fatto, tra artigiani e non, che guadagno lecito e illecito siano considerati equivalenti e non ci si fermi davanti a nulla. Questo a causa di una falsa ignoranza di ciò che è stato ordinato all'uomo e predisposto per lui di bontà d'animo nel conseguire profitti aspirando a migliorare la propria vita terrena. Apparteniamo a Dio e a Lui faremo ritorno... L'artigiano [rilegatore] rifletta, non rimandi il lavoro all'indomani, non spergiuri, ma dia ascolto all'appello alla preghiera e la compia assieme agli altri nel tempo prefissato; faccia tutto ciò con zelo, poiché i Corani, i libri di hadīt e di scienze sharicitiche che rilega lo ordinano e vietano il contrario.

\section{BIBLIOGRAFIA}

Fonti primarie

Al- ${ }^{\mathrm{c}}$ Almawī, ${ }^{\mathrm{c}} \mathrm{Abd}$ al-Bāsiț, al-Mu ${ }^{\mathrm{c}} \bar{l} d$ fì adab al-mufìd wa-l-mustafìd, ed. Marwān al- ${ }^{\mathrm{c}}$ Ațiyya, (al-Z̄āhir/Cairo: Maktabat al-țaqāfa al-dīniyya, 2003).

Al-Buhārīi, al-Ǧāmic al-ṣahịhh, ed. Muhibb al-Dīn al-Ḩațīb, et al., 4 vol. (Cairo: al-Mațba ${ }^{\mathrm{c}}$ a al-salafiyya wa-maktabuhā, 1400/1979).

Al-Ğāḥiz, [Kitāb] al-Hayawān, ed. ' Abd al-Salām Hārūn, 8 vol. (Cairo: al-Ḥalabī, $1965^{2}$ ).

Al-Ġazzālī, Abū Ḥāmid, Ihyya ${ }^{\jmath}$ culūm al-dīn, 4 vol. (Cairo: Mușțafā alBābī al-Ḥalabī, 1358/1939).

Ḥāğğī Halīfa [Haji Khalfa], Kašf al-ẓunūn c an asāmī al-kutub wa-l-funūn. Lexicon bibliographicum et encyclopcedicum, ed. G. Fluegel, 8 vol. (Leipzig, 1835-58).

Ibn Farḥūn, Ibrāhīm b. ${ }^{\mathrm{c}} \mathrm{Al} \overline{1}$, Kitāb al-Dībāğ al-mudahhab fì ma ${ }^{\mathrm{c}}$ rifat $a^{c} y \bar{a} n^{\mathrm{c}}$ ulam $\bar{a}^{\mathrm{D}}$ al-madhab, ed. Ibn Šakrūn (Cairo, 1351/1932).

Ibn Ğam ${ }^{c} a$, Muhammad b. Ibrāhīm, Tad kirat al-sāmi ${ }^{c}$ wa-l-mutakallim fì adab al- ${ }^{c} \bar{a}$ lim wa-l-mutacallim, ed. Muḥammad H. al-Nadwī (Beirut: Dār al-kutub al-'ilmiyya, 2005; rist. dell'ed. Hyderabad 1353/1934).

Ibn Ḥağar al- ${ }^{c}$ Asqalān̄i, Šihāb al-Dīn, al-Durar al-kāmina fì $a^{c} y a \bar{n}$ almì a al-tâmina, ed. Muhammad S. Ğād al-Ḥaqq (Cairo: Dār al-kutub al-ḥadīta, 1385/1966).

Ibn al-Ḥāğğ, Muḥammad al-Fāsī al- ${ }^{\mathrm{A}}$ Abdarī, al-Madhal, 4 vol. (Cairo: al-Mațba ${ }^{c}$ a al-Mișriyya bi-l-Azhar, 1348/1929; rist., Cairo: Dār al-fikr and Maktabat dār al-turāt, s.d.).

Ibn Haldūn, Muqaddimat Ibn Haldūn, ed. ${ }^{\mathrm{c}}$ Alī ${ }^{\mathrm{c}} \mathrm{Abd}$ al-Wāḥid Wāfī, (Cairo: Nahụat Mișr, 2004); Ibn Khaldûn The Muqaddimah. An Introduction to History, trad. F. Rosenthal, 3 vols. (New York: Pantheon Books, 1958). 
Ibn al-Uhuwwa, Kitāb $M a^{\mathrm{c}} \bar{a}$ lim al-qurba fì aḥkām al-ḥisba, ed. R. Levy (London: Luzac, 1937-8).

Al-Ṣafadī, Șalāḥ al-Dīn Halīl b. Aybak, Kitāb al-Wāfì bi-l-wafayāt, vol. 1, ed. H. Ritter; vol. 2, ed. S. Dedering (Wiesbaden: F. Steiner, 1962, 1974).

Al-Ša ${ }^{\mathrm{c}} \mathrm{rān} \overline{1},{ }^{\mathrm{c}} \mathrm{Abd}$ al-Wahhāb, al-Tabaqūt al-kubrā [Lawāqih al-anwār fì tabaqāt al-ahyār], 2 vol. (Cairo: al-Mațba ${ }^{\mathrm{c} a}$ al-šarafiyya, 1200 [1898]).

Al-Šayzarī, 'A Abd al-Raḥmān, Nihāyat al-rutba fì țalab al-hisba / The Book of the Islamic Market Inspector, trad. R.P. Buckley (Oxford: Oxford University Press, 1999).

Al-Subkī, Tāğ al-Dīn, Kitāb $M u^{c} \bar{\imath} d$ al-ni ${ }^{c} a m$ wa-mubìd al-niqam (Cairo: Maktabat al-Ȟānğ̄̄, 1996³).

Al-Subkī, al-Tabaqāt al-šăficiyya al-kubrāa, 6 vol. (Cairo, 1324 [1906]).

Al-Suyūṭī, Ğalāl al-Dīn, Husn al-muhạạdara fì ta rō̄h Mișr wa-l-Qāhira, ed. Muḥammad Abū 1-Faḍl Ibrāhīm, 2 vol. (Cairo: al-Ḥalabī, 1967-8).

Studi secondari

$G A L=$ Brockelmann, C., Geschichte der arabischen Litteratur (Weimar: E. Felber, 1898-1902). GAL $S=$ Erster (Zweiter, Dritter) Supplementband (Leiden: Brill, 1937-42).

$G d Q=$ Nöldeke, Th. et al., Geschichte des Qorāns, 3 vol. (Leipzig, 1909-38, repr. Hildesheim: G. Olms, 1961).

${ }^{\mathrm{c}}$ Abd al-Rāziq, A., 'La hisba et le muhtasib en Égypte au temps des Mamlūks', Annales Islamologiques, 13 (1977), 116-78.

Ashtor, E., Storia sociale ed economica del Vicino Oriente nel Medioevo, (trad. it., Torino: Einaudi, 1982).

Al-Bāšā, H.H., al-Funūn al-islāmiyya wa-l-waz $\bar{a}^{\supset}$ if c alā l-ātāar al- ${ }^{c}$ arabiyya 4 vol. (Cairo: Dār al-nahḍa al- ${ }^{c}$ arabiyya, 1965).

Blair, S.S., Islamic Calligraphy (Cairo: The American University Press, 2006).

Bloom, J.M. Paper before Print. The History and Impact of Paper in the Islamic World (New Haven-London: Yale University Press, 2001).

Buckley, R.P., 'The muhtasib', Arabica 39 (1992), 59-117.

Canova, G., 'Critical Attitudes toward Arabic Folk Epics', Eurasian Studies, 4 (2005), 29-40.

__ 'Libri e artigiani del libro: le raccomandazioni di Tāj al-Dīn alSubkī (XIV secolo)', in Loquentes linguis. Studi linguistici e orientali in onore di Fabrizio A. Pennacchietti, ed. P.G. Borbone, A. Mengozzi, M. Tosco (Wiesbaden: Harrassowitz, 2006, 159-66). 
Canova, G., 'Libri, documenti e materiale scrittorio nell'onirocritica araba', in D. Bredi et al. (eds), Scritti in onore di Biancamaria Scarcia Amoretti (Roma, Edizioni Q, 2008), 1: 291-308.

Capezzone, L., La trasmissione del sapere nell'Islam medievale (Roma: Jouvence, 1998).

Cassarino, M., 'Ancora sulla definizione di $a d a b$ ', in E. Creazzo et al. (eds), Racconti senza fine per Antonio Pioletti, (Soveria Mannelli: Rubbettino, 2011), 47-67.

Cook, M., Commanding Right and Forbidding Wrong in Islamic Thought (Cambridge: Cambridge University Press, 2000).

Déroche, Fr. Manuel de codicologie des manuscrits en écriture arabe (Paris: Bibliothèque nationale de France, 2000).

Déroche, Fr. e Fr. Richard (dir.), Scribes et manuscrits du Moyen-Orient (Paris: Bibliothèque nationale de France, 1997).

Eche, Y. [Yūsuf al-' ${ }^{-}$Išš], Les Bibliothèques arabes publiques et semipubliques en Mésopotamie, en Syrie et en Égypte au Moyen Age (Damas: I.F.D., 1967).

Fierro, M., 'The Treatises against Innovations (Kutub al-bidac $)^{c}$, Der Islam, 69 (1992), 204-46.

Gabrieli, F., 'Adab', in $E I^{2}, 1: 180-1$.

Gacek, A., The Arabic Manuscript Tradition. A Glossary of Technical Terms and Bibliography (Leiden-Boston-Köln: Brill, 2001. Supplement, 2008).

- 'Technical practices and recommendations recordered by classsical and post-classical Arabic scholars concerning the copying and correction of manuscripts', in F. Déroche (ed.), Les Manuscrits du Moyen-Orient. Essais de codicologie et de paléographie (IstanbulParis, Institut Français d'Études Anatoliennes: Bibliothèque nationale, 1989), 51-60.

_- Arabic Manuscripts. A Vademecum for Readers (Leiden-Boston: Brill, 2009).

Gaudefroy-Demombynes, 'Sur quelques manuels de hisba', Journal Asiatique, 230 (1938), 449-57.

Ghabin, A., Hisba, Arts and Crafts in Islam (Wiesbaden: Harrassowitz, 2009).

Ghersetti, A., 'L'utilità della scrittura e la lode del libro: testimonianze di alcuni scrittori arabi', Annali di Ca' Foscari, 25, (1994), 67-76.

Al-Hazīmī, N., Haraq al-kutub fí l-turāt al- ${ }^{c}$ arabī (Köln: Kamel Verlag, 2003). 
Grohmann, A., 'Bibliotheken und Bibliophilen im islamischen Orient', in Festschrift der Nationalbibliothek in Wien (Wien: Österreichische Staatsdruckerei, 1926), 431-42.

—, Arabische Paläographie, 2 vol. (Wien: Böhlaus, 1967).

Grunebaum, G.E. von, 'The Structure of the Muslim Town', in Islam. Essays in the Nature and Growth of a Cultural Tradition, The American Anthropologist, Memoir no. 81 (1955), 141-58.

Al-Ğubūrī, Y.W., al-Kitāb fì l-ḥad̄ara al-islāmiyya (Beirut, Dār al-Ġarb al-Islāmī, 1998).

Hourani, A.H. e S.M. Stern (eds), The Islamic City. A Colloquium (Oxford: B. Cassirer, 1970).

Irwin, R., Islamic Art (London: Laurence King, 1997).

Jayyusi, S.K., The City in the Islamic World (Leiden-Boston: Brill, 2008).

Kremer, von A., Kulturgeschichte des Orients unter den Chalifen, repr. Aalen: Scientia Verlag, 1966 [Wien 1877].

Lapidus, I.M., Muslim Cities in the Later Middle Ages (Cambridge, Mass.: Harvard University Press, 1967).

Lewis, B., 'An Epistle on Manual Crafts', Islamic Culture, 17 (1943), $142-51$.

Massignon, L., 'Les Corps de métiers et la cité islamique', in Opera minora, I, Paris: P.U.F.,

Pedersen, J., The Arabic Book, (ed. orig. Den Arabiske bog, Copenhagen, 1946; trad. ingl., R. Hillenbrand (ed.), Princeton: Princeton University Press, 1984).

Pinto, O., 'Le biblioteche degli Arabi nell'età degli Abbassidi', La Bibliofilia, 30 (1928), 139-165.

Quatremère, É., 'Mémoire sur le goût des livres chez les Orientaux', Journal Asiatique, III ser., 6 (1838), 35-78.

Raymond, A. and G. Wiet, Les Marchées du Caire. Traduction annotée du texte de Maqrīzī, 2 vol. (Le Caire: I.F.A.O., 1979).

Rispler, V., 'Toward a New Understanding of the Term bid'a', Der Islam 68 (1991), 320-8.

Ritter, H., 'Ein arabisches Handbuch der Handelswissenschaft', Der Islam 7 (1917), 1-91.

Rosenthal, F., The Technique and Approach of Muslim Scholarship (Roma: Pontificium Institutum Biblicum, 1947).

_- Significant uses of Arabic writing, in Id., Four essays on art and literature in Islam (Leiden, E.J. Brill, 1971).

_ _ " "Of Making Many Books there is no End:" The Classical Muslim View', in G.N. Atiyeh (ed.), The Book in the Islamic World (Albany: State University of New York Press, 1995), 33-55. 
Sadan, J., 'Nouveaux documents sur scribes et copistes', Revue des études Islamiques, 45 (1977), 41-87.

- 'Genizah and Genizah-like practices in Islamic and Jewish traditions', Bibliotheca orientalis, 43 (1986), 36-58.

Salibi, K.S., 'The Banū Jamā̄a: A Dynasty of Shāficite Jurists', Studia Islamica, 9 (1958), 97-109.

Sauvaget, J. [Cl. Cahen], Introduction à l'histoire de l'Orient musulman. Éléments de bibliographie (Paris: Adrien-Maisonneuve, 1961).

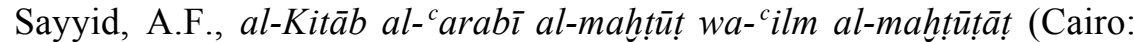
al-Dār al-mișriyya al-lubnāniyya, 1997).

Scarcia Amoretti, B., Un altro Medioevo. Il quotidiano nell'Islam (Roma-Bari: Laterza, 2001), cap. III.

Schacht, J., An Introduction to Islamic Law (Oxford: Clarendon Press, 1964).

Schacht, J. [C.E. Bosworth], 'al-Subkī', in Encyclopédie de l'Islam ${ }^{2}$ (Leiden: Brill, 1998), ix, 776-8.

Serjeant, R.B. (ed.), The Islamic City (Paris: UNESCO, 1980).

Shatzmiller, M., Labour in the Medieval Islamic World (Leiden-New York-Köln: Brill, 1994).

Touati, H., Biblioteche di saggezza. Libro e collezionismo nell'Islam (ed. or. L'Armoire à sagesse. Bibliothèques et collections en Islam, Paris 2003; trad. it., Milano: S. Bonnard, 2006).

Vadet, J.-C., 'Ibn al-Hāājdj', in Encyclopédie de l'Islam ${ }^{2}$ (Leiden: Brill, 1971), iii, 803.

Vercellin, G., 'Hisba: Religious Duty or Practical Job? Some Considerations on an Islamic Institution between Morals and Markets', Annali di Ca' Foscari, 29 (1998), 67-96.

Winkler, H.A., Siegel und Charaktere in der muhammedanischen Zauberei (Berlin-Leipzig: W. de Gruyter, 1930).

Al-Ziriklī, Huayr al-Dīn, al-A lām. Qāmūs tarāăgim li-ašhar al-riğāal wa-lnis $\bar{a}^{\supset}$ min al- ${ }^{c}$ arab wa-l-mustac ${ }^{c}$ ribìn wa-l-mustašriqìn, 13 vol. (Beirut: Dār al- ${ }^{c}$ ilm li-1-malāyīn, $1980^{5}$ ). 